\title{
FAISCEAUX AMPLES SUR LES ESPACES ANALYTIQUES \\ BY
}

VINCENZO ANCONA

\begin{abstract}
The following result is established. Let $f: X \rightarrow Y$ be a morphism between two compact complex spaces and $\mathcal{L}$ a weakly positive invertible sheaf on $X$; then for suitable $n f_{*} \mathcal{L}^{n}$ is weakly positive on $Y$.

It follows that Moišezon spaces can be characterized via weakly positive coherent sheaves. Moreover, a problem posed by Grauert on the exceptional subspaces of complex spaces can be solved.
\end{abstract}

Introduction. Parmi les nombreuses définitions de positivité pour les faisceaux cohérents sur un espace analytique compact l'une des plus naturelles est sans doute celle de positivité faible donnée (implicitement) par Grauert [12].

Dans ce travail on trouve que la notion de faisceau faiblement positif est équivalente à celle de amplitude (cohomologique). On peut donc prouver que les images directes de puissances symétriques convenables d'un faisceau faiblement positif (en particulier, d'un faisceau inversible ample) sont faiblement positives (Théorème 3.1).

Le Théorème 3.1 peut être aussi considéré comme un théorème d'existence de faisceaux faiblement positifs non localement libres. En outre il permet de caractériser les espaces de Moišezon comme les espaces analytiques compacts qui portent un faisceau faiblement positif de rang 1 (Théorème 3.6), et aussi de répondre à une conjecture de Grauert [12] sur les sous-espaces exceptionnels d'un espace analytique (Théorème 3.7).

Tous ces résultats ont un analogue "relatif". Comme conséquence, on peut établir un théorème d'existence des contractions d'espaces analytiques, qui généralise celui de Fujiki [11, Théorème 4.1].

0. Notations et conventions. Dans tout le travail, on suppose que les espaces analytiques (complexes) considérés soient séparés, réunions dénombrables de compacts, mais non nécessairement réduits; que les espaces algébriques et les espaces algébriques formels, ainsi que leurs morphismes, soient séparés. On renvoie à [6, 19] pour la définition et les propriétés des espaces algébriques.

Si $\mathcal{E}$ est un faisceau cohérent sur un espace analytique ou algébrique, on dénote par $S^{n} \mathcal{E}$ la $n$-ième puissance tensorielle symétrique de $\mathcal{E}$, et par $L(\mathcal{E})$ l'espace fibré linéaire associé à $\mathcal{E}[2,10]$; on a $L(\mathcal{E})=$ Specan $S \mathcal{E}, S \mathcal{E}=\bigoplus_{n \geqslant 0} S^{n} \mathcal{E}$ étant l'algèbre symétrique de $\mathcal{E}$.

Received by the editors February 18, 1981.

1980 Mathematics Subject Classification. Primary 32J99; Secondary 32C35, 32L99.

Key words and phrases. Faisceau coherent faiblement positif, ample, cohomologiquement ample, espace de Moišezon, sous-espace exceptionnel, fibré normal, contraction. 
Soient $X$ un espace analytique, $A \subset X$ un sous-espace analytique fermé, $f: A \rightarrow A^{\prime}$ un morphisme propre surjectif sur un autre espace analytique $A^{\prime}$. On dit qu'on peut contracter $X$ le long de $f$ s'il existe un espace analytique $X^{\prime}$, un plongement fermé $j$ : $A^{\prime} \rightarrow X^{\prime}$, un morphisme propre surjectif $g: X \rightarrow X^{\prime}$ tel que $g_{\mid A}=j \circ f, g^{-1}\left(j\left(A^{\prime}\right)\right)=A$ et $g$ induit un isomorphisme de $X \backslash A$ sur $X^{\prime} \backslash j\left(A^{\prime}\right)$ (donc $g$ est une modification); on écrira $g:(A, X) \rightarrow\left(A^{\prime}, X^{\prime}\right)$. On dit que $X^{\prime}$ est la contraction de $X$ le long de $f$. Une définition semblable vaut pour les espaces algébriques.

Si $p: X \rightarrow Y$ est une application d'ensembles et $V$ est un sous-ensemble de $Y$, on pose par définition $X_{\mid V}=p^{-1}(V)$.

Enfin si $X$ est un espace analytique ou algébrique, on note $\operatorname{Coh}(X)$ l'ensemble des classes d'isomorphisme de faisceaux cohérents sur $X$.

1. Espaces algèbriques sur une algébre de Stein. Soit $S$ un espace algébrique noethérien sur un corps, non nécessairement de type fini sur celui-ci. On a le théorème d'existence des contractions suivant:

ThEOREME 1.1. Soient $X$ un espace algébrique de type fini sur $S, Y \subset X$ un sous-espace algébrique fermé, $g \subset \vartheta_{X}$ l'idéal de $Y, g: Y \rightarrow Y^{\prime}$ un $S$-morphisme propre surjectif sur un $S$-espace algébrique de type fini $Y^{\prime}$. On suppose

(i) Pour tout faisceau cohérent $\mathcal{F}$ sur $Y$ il existe un entier $n_{0}$ tel que si $n \geqslant n_{0}$ on ait

$$
R^{1} g_{*}\left(\mathscr{F} \otimes S^{n}\left(J / J^{2}\right)\right)=0 .
$$

(ii) Pour tout entier $n$ l'application de faisceaux sur $Y^{\prime}$

$$
g_{*}\left(\theta_{X} / g^{n}\right) \times_{g_{*} \theta_{Y}} \theta_{Y^{\prime}} \rightarrow \theta_{Y^{\prime}}
$$

est surjective.

On peut alors contracter $X$ le long de $g$.

PreUve. La condition (i) implique que pour tout faisceau cohérent $\mathscr{F}$ sur $Y$ on a $R^{1} g_{*}\left(g^{n} \widetilde{F} / g^{n+1} \mathscr{F}\right)=0$ pour $n \gg 0$ [21, Proposition 1]. D'après [7, Théorème 6.2], il existe une $S$-contraction formelle $\mathfrak{X} \rightarrow \mathfrak{X}^{\prime}$ où $\mathfrak{X}$ est le complété formel de $X$ le long de $Y$. La conclusion résulte du théorème d'existence des contractions [32, Théorème 4.1].

Remarque. Si $S$ est de type fini sur un corps, le théorème est dû à M. Artin (voir [21, Corollaire 1]).

Soient $V$ un espace de Stein, $K \subset V$ un compact de Stein de $V$, i.e. un compact semi-analytique ayant un système fondamental de voisinages ouverts de Stein. Posons $S_{V}=\operatorname{Spec} \Gamma\left(V, \theta_{V}\right), S_{K}=\operatorname{Spec} \Gamma\left(K, \theta_{V}\right)$; ce sont deux schémas affines sur C; $S_{K}$ est noethérien.

Si $Z$ est un $S_{V}$-espace algébrique de type fini, on peut lui associer un espace analytique $Z^{\text {an }}$ sur $V$; on obtient ainsi un foncteur de la catégorie des $S_{V}$-espaces algébriques de type fini dans celle des espaces analytiques sur $V$. On dit qu'un espace analytique $X$ sur $V$ est algébrisable s'il est $Y$-isomorphe à $Z^{\text {an }}$, où $Z$ est un $S_{V^{-}}$ espace algébrique de type fini, qu'un faisceau analytique cohérent $\mathscr{F}$ sur $X$ est algébrisable s'il est de la forme $\mathcal{G}^{\text {an }}, \mathcal{G}$ étant un faisceau algébrique cohérent sur $Z$; qu'un $Y$-morphisme $f: X \rightarrow X^{\prime}$ dans un autre espace analytique algébrisable $X^{\prime}$, avec 
$X^{\prime} \simeq Z^{\prime \text { an }}$, est algébrisable s'il est de la forme $g^{\text {an }}$, où $g: Z \rightarrow Z^{\prime}$ est un $S_{V}$-morphisme d'espaces algébriques.

Si $Z$ est un $S_{V}$-espace algébrique, on pose $Z_{K}=Z \times_{S_{V}} S_{K}$. On a le théorème de comparaison suivant ("GAGA relatif").

THEORÈmE 1.2. Soient $V$ un espace de Stein, $K \subset V$ un compact de Stein, $Z$ un $S_{V}$-espace algébrique propre de type fini, $p: Z \rightarrow S_{V}$ le morphisme structural. Alors

(i) Pour tout faisceau cohérent $\mathscr{F}$ sur $Z$ et tout entier n, les morphismes naturels

$$
\left(R^{n} p_{*} \mathscr{F}\right)^{\text {an }} \rightarrow R^{n} p_{*}^{\text {an }} \mathscr{F}^{\text {an }}
$$

sont des isomorphismes.

(ii) L'application naturelle

$$
\operatorname{Coh}\left(Z_{K}\right) \rightarrow \lim _{U} \operatorname{ind} \operatorname{Coh}\left(Z^{\text {an }}{ }_{\mid U}\right)
$$

est bijective (ici $U$ parcourt un système fondamental de voisinages ouverts de $K$ dans V).

La preuve de ce théorème se trouve dans $[9,15]$ dans le cas où $Z$ est un schéma, et peut se traduire mot par mot au cas des espaces algébriques.

Si $X$ est un espace analytique, dénotons par $\mathfrak{M}(X)$ le corps des fonctions méromorphes globales sur $X$.

Soit $f: X \rightarrow Y$ un morphisme propre d'espaces analytiques. On dit que $X$ est $f$-Moišezon [3] si pour tout $y \in Y$ il existe un voisinage ouvert de Stein $V$ de $y$ dans $Y$ tel que:

(1) $\left(X_{\mid V}\right)_{\text {red }}$ a un nombre fini de composantes irréductibles $X_{1}, \ldots, X_{r}$;

(2) si $Y_{j}=f\left(X_{j}\right)(j=1, \ldots, r)$ on a

$$
t\left(X_{j}, Y_{j}\right)=\operatorname{dim} X_{j}-\operatorname{dim} Y_{j}
$$

où $t\left(X_{j}, Y_{j}\right)$ est le degré de transcendance de $\mathfrak{M}\left(X_{j}\right)$ sur $\mathfrak{M}\left(Y_{j}\right)$.

Remarquons qu'en tout cas on a $t\left(X_{j}, Y_{j}\right) \leqslant \operatorname{dim} X_{j}-\operatorname{dim} Y_{j}$ [5, Théorème 10.9].

Dans [3] nous avons démontré le théorème suivant.

THEORÈm 1.3. Soit $f: X \rightarrow Y$ un morphisme propre d'espaces analytiques tel que $X$ soit f-Moišezon. Pour tout $y \in Y$ il existe un voisinage ouvert de Stein $V$ de $y$ et un $S_{V}$-espace algébrique $Z$, propre et de type fini, tel que $Z^{\text {an }}$ soit $V$-isomorphe à $X_{\mid V}$.

2. Faisceaux relativement amples sur les espaces analytiques. Soient $f: X \rightarrow Y$ un morphisme propre d'espaces analytiques, $\mathcal{E}$ un faisceau cohérent sur $X$. Pour tout faisceau cohérent $\mathscr{F}$ sur $X$ et tout entier $n$ on pose $\mathscr{F}(n)=\mathscr{F} \otimes S^{n} \mathscr{E}$.

Defrinition 2.1. On dit que $\mathcal{E}$ est f-ample si pour tout faisceau cohérent $\mathscr{F}$ sur $X$ et tout compact $K \subset Y$ il existe un entier $n_{0}$ tel que le morphisme naturel de faisceaux

$$
f^{*} f_{*} \mathscr{F}(n) \rightarrow \mathscr{F}(n)
$$

soit surjectif au dessus de $K$ pour $n \geqslant n_{0}$.

DEFINITION 2.2. On dit que $\mathcal{E}$ est cohomologiquement f-ample si pour tout faisceau cohérent $\mathscr{F}$ sur $X$ et tout compact $K \subset Y$ il existe un entier $n_{0}$ tel que $R^{q} f_{*} \mathscr{F}(n)_{\mid K}=0$ pour $n \geqslant n_{0}$ et $q \geqslant 1$. 
DEFINITION 2.3. Si $X=\operatorname{Supp} \mathcal{E}$, on dit que $\mathcal{E}$ est faiblement f-positif si pour tout $y \in Y$ il existe un voisinage ouvert $V$ de y dans $Y$ tel que $L(\mathcal{E})_{\mid V}$ peut être contracté le long de $f$ (on identifie $X$ à la section nulle de $L(\mathcal{E})$ ). En général on dit que $\mathcal{E}$ est faiblement $f$-positif si sa restriction à Supp $\mathcal{E}$ est faiblement $f_{0}$-positif, $f_{0}=f_{\mid \text {Supp } \mathscr{E}}$.

$\mathcal{E}$ est faiblement $f$-positif si $L(\mathcal{E})$ est faiblement négatif relativement à $f$, au sens de [18]. En particulier si $Y$ est réduit à un point, ceci signifie que $L(\mathcal{E})$ est faiblement négatif au sens de Grauert [12].

On peut caractériser les faisceaux faiblement $f$-positifs au moyen des morphismes 1-convexes (voir $[1,2,18])$.

Si $Y$ est un point, on dit respectivement que $\mathcal{E}$ est ample, cohomologiquement ample, faiblement positif.

Soient $P=\mathbf{P}(\mathcal{E})$ le fibré projectif associé à $\mathcal{E}[27$, Exposé $\mathrm{XII}], p: P \rightarrow X$ la projection, $\mathcal{L}=\mathcal{O}_{P}(1)$ le faisceau inversible canonique. On a

LEMME 2.4. Il existe pour tout entier positif $n$ un homomorphisme naturel

$$
\alpha_{n}: \mathscr{F} \otimes S^{n} \mathcal{E} \rightarrow p_{*}\left(p^{*} \mathscr{F} \otimes \mathcal{E}^{n}\right) .
$$

De plus, pour tout compact $K \subset X$ il existe un entier $n_{0}$ tel que pour $n \geqslant n_{0} \alpha_{n}$ est un isomorphisme au dessus de $K$.

Preuve. Puisque on cherche des homomorphismes naturels, il est clair que le problème est local sur $X$. On peut donc supposer que $K$ soit un compact de Stein de $X$, et qu'il existe deux faisceaux cohérents $\mathcal{E}^{\prime}, \mathcal{F}^{\prime}$ sur $S_{K}=\operatorname{Spec} \Gamma\left(K, \vartheta_{X}\right)$ tels que $\mathcal{E}^{\prime a n}=\mathcal{E}, \mathcal{F}^{\prime a n}=\mathscr{F}$. Soient $P^{\prime}=\mathbf{P}\left(\mathcal{E}^{\prime}\right)$ le fibré projectif (algébrique) associé à $\mathcal{E}^{\prime}, p^{\prime}$ : $P^{\prime} \rightarrow S_{K}$ la projection; $\mathcal{L}^{\prime}=\mathcal{O}_{P^{\prime}}(1)$. Alors on a $P^{\prime a n} \simeq P, p^{\prime a n}=p, \mathcal{L}^{\prime a n}=\mathcal{L}$. D'après [14, II, 3.3.2 et III, 2.3.1], il existe des homomorphismes naturels $\alpha_{n}^{\prime}: \mathscr{F}^{\prime} \otimes S^{n} \mathcal{E}^{\prime} \rightarrow p_{*}^{\prime}$ $\left(p^{\prime * \mathscr{F}^{\prime}} \otimes \mathcal{L}^{\prime n}\right)$ qui sont des isomorphismes pour $n \gg 0$; alors les $\alpha_{n}=\alpha_{n}^{\text {an }}$ sont les homomorphismes cherchés.

Posons $\pi=f \circ p$.

Proposition 2.5. Les conditions suivantes sont équivalentes.

(i) $\varepsilon$ est $f$-ample,

(ii) E est cohomologiquement f-ample,

(iii) Lest $\pi$-ample,

(iv) L est cohomologiquement $\pi$-ample.

DEmonstration. L'équivalence de (iii) et (iv) est bien connue $[16,11]$. On peut prouver les implications (iv) $\Rightarrow$ (ii) et (i) $\Rightarrow$ (iii) de façon analogue à la preuve de la Proposition 3.2 de [15], compte tenu du Lemme 2.4.

Il reste à prouver l'implication (ii) $\Rightarrow$ (i). Si $x \in X$, on note $\mathfrak{m}_{x}$ l'idéal cohérent de $\vartheta_{X}$ des germes de sections nulles en $x$. Pour tout faisceau cohérent $\mathscr{F}$ sur $X$ et pour tout entier $n$ on trouve une suite exacte

$$
0 \rightarrow \mathcal{H} \rightarrow \mathfrak{m}_{x} \otimes \mathscr{F}(n) \rightarrow \mathscr{F}(n) \stackrel{v}{\rightarrow} \theta_{X} / \mathfrak{m}_{x} \otimes \mathscr{F}(n) \rightarrow 0
$$

où $\mathcal{H}$ est un faisceau cohérent dont le support est $\{x\}$ (cf. [2,pp. 57-58]). On a $R^{2} f_{*} \mathcal{H C}=0$, donc pour tout compact $K \subset Y$, si $n$ est assez grand on trouve $R^{1} f_{*}(\operatorname{Ker} v)=0$ sur $K$, d'où la conclusion. 
Corollaire 2.6. Si pour tout faisceau cohérent $\mathscr{F}$ sur $X$ et tout compact $K \subset Y$ il existe un entier $n_{0}$ tel que $R^{1} f_{*}\left(\mathscr{F} \otimes S^{n} \mathcal{E}\right)_{\mid K}=0$ pour $n \geqslant n_{0}$, $\mathcal{E}$ est cohomologiquement f-ample.

Corollaire 2.7. E est f-ample si et seulement si $\mathcal{E}_{\mathrm{red}}$ est $f_{\mathrm{red}}$-ample $\left(f_{\mathrm{red}}: X_{\mathrm{red}} \rightarrow\right.$ $\left.Y_{\text {red }}\right)$.

En effet, c'est bien connu pour les faisceaux inversibles.

Proposition 2.8. Si E est cohomologiquement f-ample, Supp E est f-Moišezon.

On peut supposer $X=\operatorname{Supp} \mathcal{E}$. Alors $\pi$ est projectif (localement par rapport à $Y$ ) d'après 2.5. Comme $p$ est surjectif, la proposition descend du Théorème 2.5 de [3].

On va maintenant prouver:

ThEorÈme 2.9. Soient $f: X \rightarrow Y$ un morphisme propre d'espaces analytiques, $\mathcal{E}$ un faisceau cohérent sur $X, P=\mathbf{P}(\mathcal{E}), p: P \rightarrow X$ la projection, $\mathcal{L}=\vartheta_{P}(1)$. Les conditions suivantes sont équivalentes.

(i) Lest $(f \circ p)$-ample,

(ii) $\mathcal{E}$ est $f$-ample,

(iii) E est cohomologiquement f-ample,

(iv) $\mathcal{E}$ est faiblement f-positif.

Preuve. On peut supposer $X=\operatorname{Supp} \mathcal{E}$. Il ne reste à prouver que l'équivalence entre (iii) et (iv). L'implication (iv) $\Rightarrow$ (iii) est prouvée dans [2, Théorème 3.4]. On va prouver l'implication (iii) $\Rightarrow$ (iv). Supposons donc $\mathscr{E}$ cohomologiquement $f$-ample. D'après la Proposition 2.8, $X$ est $f$-Moišezon. Soit $y \in Y$; il existe un voisinage ouvert de Stein $V$ de $y$, relativement compact, et un $S_{V}$-espace algébrique propre et de type fini $Z$ tel que $Z^{\text {an }}$ soit $V$-isomorphe à $X_{\mid V}$ (Théorème 1.3).

Soient $T=L(\mathcal{E}), 9$ i'idéal cohérent de $\theta_{T}$ qui définit le plongement $i: X \rightarrow T$ de $X$ comme section nulle. On a un isomorphisme naturel $\mathscr{G} / \mathscr{G}^{2} \simeq \mathcal{E}$ [2, Remarque, p. 51] donc $g / g^{2}$ est cohomologiquement $f$-ample. D'après le Théorème 1.2 la restriction de $\mathcal{E}$ à $X_{\mid V}$ est de la forme $\mathcal{G}^{\text {an }}, \mathcal{G}$ étant un faisceau cohérent sur $Z$. Soient $g: Z \rightarrow S_{V}$ le morphisme structural, $W=L(\mathcal{G}), j: Z \rightarrow W$ le plongement comme section nulle, $q$ : $W \rightarrow Z$ la projection, $g$ l'idéal de $\theta_{W}$ qui définit le plongement $j$. On a $W^{\text {an }} \simeq T$, $\left(g / g^{2}\right)^{\text {an }} \simeq \mathscr{g} / \mathscr{G}^{2}$. D'autre part, $W$ est un $S_{V}$-espace algébrique de type fini au moyen du morphisme $g \circ q$.

Soit $K$ un compact de Stein, voisinage de $y, K \subset V$, et prenons $S_{K}$ comme espace de base, au moyen du foncteur $\times_{S_{V}} S_{K}$; nous continuons à écrire $Z$ au lieu de $Z_{K}, g$ au lieu de $g_{K}$ et ainsi de suite. D'après de Théorème 1.2(i), pour tout faisceau cohérent $\mathscr{F}$ sur $Z$ on a $R^{1} g_{*}\left(\mathscr{F} \otimes S^{n}\left(\mathscr{G} / \mathscr{f}^{2}\right)\right)=0$ pour $n \gg 0$. D'autre part, comme il existe une projection de $W$ sur $Z$, les applications de faisceaux $g_{*}\left(\theta_{W} / g^{n}\right) \times_{g_{*} \theta_{Z}}$ $\vartheta_{S_{K}} \rightarrow \vartheta_{S_{K}}$ sont surjectives pour tout $n>0$. D'après le Théorème 1.1 on peut contracter $W$ le long de $g$ à un $S_{K}$ espace algébrique de type fini $Q$. Il est alors clair que si $U=K^{0}, Q_{\mid U}^{\text {an }}$ est une contraction de $L(\mathcal{E})_{\mid U}$ de long de $f$.

Remarque. Dans le cas où $\mathcal{E}$ est localement trivial, le théorème été prouvé, avec des méthodes différentes, par M. Schneider [26]. 
Corollaire 2.10. Soit $y \in Y$ tel que le faisceau restriction de $\mathcal{E}$ à la fibre $f^{-1}(y)$ soit faiblement positif. Alors il existe un voisinage ouvert $U$ de y dans $Y$ tel que $\mathcal{E}_{f^{-1}(U)}$ soit faiblement f-positif.

Démonstration. D'après le théorème, on se ramène au cas où $\mathcal{E}$ est un faisceau inversible. Soient $y \in Y, T_{0}=f^{-1}(y), T_{n}$ le $n$-ième voisinage infinitésimal de $T_{0}$ dans $X$ ( $n$ entier non négatif). D'après (2.7), tous les $T_{n}$ sont des variétés projectives; d'après un théorème de Kuhlmann [20] le morphisme $f$ est projectif au voisinage de $y$, donc relativement algébrisable. On conclut par GAGA et [14, Théorème III, 4.7.1].

Corollaire 2.11. Soient $X$ un espace analytique compact, $\mathcal{E}$ un faisceau cohérent sur $X$. Les propriétés suivantes sont équivalentes.

(i) E est ample,

(ii) E est cohomologiquement ample,

(iii) $\mathcal{E}$ est faiblement positif.

3. Images directes de faisceaux faiblement positifs. Soient $f: X \rightarrow Y$ un morphisme d'espaces analytiques compacts, et $\mathcal{E}$ un faisceau faiblement positif sur $X$. En général, $f_{*} \mathcal{E}$ n'est pas faiblement positif sur $Y$ (voir un exemple très simple dans [16]). Cependant, on a

THEOREME 3.1. Soient $f: X \rightarrow Y$ un morphisme $d$ 'espaces analytiques compacts, $\mathcal{E}$ un faisceau faiblement positif sur $X$. Il existe un entier positif $n_{0}$ tel que $f_{*}\left(S^{n_{0} n} \mathcal{E}\right)$ soit faiblement positif sur $Y$ pour tout $n \geqslant 1$.

On a besoin de quelques lemmes préliminaires.

Soit $\mathscr{F}$ un faisceau cohérent sur un espace analytique compact $Y$. On dit que $\mathscr{F}$ est engendré par ses sections globales si pour tout $y \in Y$ l'application naturelle

$$
\Gamma(Y, \mathscr{F}) \rightarrow \mathscr{F}_{y} / \mathrm{m}_{y} \mathscr{F}_{y}
$$

est surjective ( $m_{y}$ désigne soit l'idéal maximal de $\vartheta_{Y, y}$, soit l'idéal cohérent de $\vartheta_{Y}$ des germes de sections nulles en $y$ ).

Nous dirons que $\mathcal{F}$ sépare les points de $Y$ si pour tout couple de points distincts $y_{1}$, $y_{2} \in Y$ l'application naturelle

$$
\Gamma(Y, \mathscr{F}) \rightarrow \mathscr{F}_{y_{1}} / \mathfrak{m}_{y_{1}} \mathscr{F}_{y_{1}} \oplus \mathscr{F}_{y_{2}} / \mathfrak{m}_{y_{2}} \mathscr{F}_{y_{2}}
$$

est surjective (en particulier, $\mathscr{F}$ est engendré par ses sections globales).

LEMME 3.2. Soient $f: X \rightarrow Y$ un morphisme fini $d$ 'espaces analytiques compacts, $\mathcal{L}$ un faisceau inversible ample sur $Y$. Alors $f^{*} \mathcal{L}$ est ample sur $X$.

Le lemme, bien connu, est une conséquence du fait que si $\mathcal{G}$ est un faisceau cohérent sur $X$ on a pour tout $n \geqslant 1$ un isomorphisme naturel $f_{*} \mathcal{G} \otimes \complement^{n} \tilde{\rightarrow}$

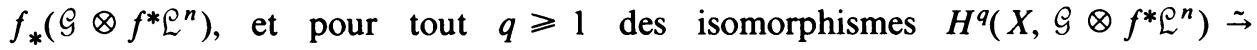
$H^{q}\left(Y, f_{*}\left(\mathcal{G} \otimes f^{*} \mathcal{L}^{n}\right)\right)(f$ étant fini $)$.

LEMME 3.3. Soient $Y$ un espace analytique compact et $\mathcal{L}$ un faisceau inversible sur $Y$ engendré par ses sections globales. On suppose que pour tout sous-espace fermé $Z$ de $Y$ tel que $\mathcal{L}_{\mid Z}$ soit trivial on ait $\operatorname{dim} Z=0$. Alors $\mathcal{L}$ est ample. 
Preuve (cfr. [30]). Les sections globales de $\mathcal{L}$ définissent un morphisme $r$ de $Y$ dans espace projectif $\mathbf{P}^{N}$, tel que $\mathcal{L}=r^{*} \mathcal{H}$ où $\mathcal{H}$ est un faisceau inversible ample sur $\mathbf{P}^{N}$. Comme la restriction de $\mathcal{E}$ à chaque fibre de $r$ est triviale, il s'ensuit que $r$ est fini; la conclusion résulte du Lemme 3.2.

LEMME 3.4. Soient $Y$ un espace compact, $\mathscr{F}$ un faisceau cohérent sur $Y$. Si F sépare les points de $Y$ il est faiblement positif.

Soient $P=\mathbf{P}(\mathcal{F}), \mathcal{L}=\vartheta_{P}(1), p: P \rightarrow Y$ la projection. Comme $\mathscr{F}$ est engendré par ses sections globales, on a sur $Y$ une surjection de faisceaux $\vartheta_{Y}^{q+1} \rightarrow \mathcal{F} \rightarrow 0$ d'où un plongement fermé $P \stackrel{i}{\rightarrow} Y \times \mathbf{P}^{q}$, tel que $\mathcal{L}=i^{*} \mathcal{\vartheta}_{Y \times \mathbf{P}^{q}}(1)$. Il s'ensuit que $\mathcal{L}$ est en-

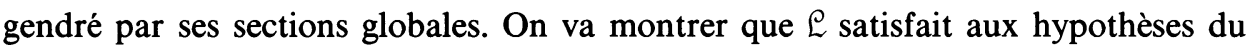
Lemme 3.3. Soit $Z$ un sous-espace fermé de $P$ tel que $\varrho_{Z Z}$ soit trivial. Comme la restriction de $\mathcal{L}$ à toute fibre de $p$ est ample, il s'ensuit que $Z$ coupe chacune de ces fibres en un nombre fini de points. Donc si $\operatorname{dim} Z>0$, il existe une composante irréductible $T \operatorname{de} Z, \operatorname{dim} T>0$, et deux points $x_{1}, x_{2} \in T$ tels que $y_{1}=p\left(x_{1}\right) \neq y_{2}=$ $p\left(x_{2}\right)$.

Comme $\mathcal{L}$ est $p$-ample, il existe une section $t_{1} \in \Gamma\left(p^{-1}\left(y_{1}\right), \mathcal{L}^{k}\right)(k$ entier convenable) tel que son image $t_{1}\left(x_{1}\right) \in \mathcal{L}_{x_{1}}^{k} / \mathfrak{m}_{x_{1}} \mathcal{L}_{x_{1}}^{k}$ soit non nulle. D'autre part $t_{1}$ donne un élément $\tilde{t}_{1} \in\left(p_{*} \varrho^{k}\right)_{y_{1}}$.

Quitte à remplacer $k$ par un multiple, on peut supposer que $p_{*} \mathcal{L}^{k}=S^{k} \mathscr{F}$ (Lemme 2.4). Comme $\mathscr{F}$ sépare les points de $Y$, il en est ainsi de $S^{k} \mathscr{F}$, donc il existe $\tilde{t} \in \Gamma\left(Y, S^{k} \mathscr{F}\right)=\Gamma\left(Y, p_{*} \mathcal{L}^{k}\right)$ tel que $\tilde{t}-\tilde{t}_{1}=0 \bmod m_{y_{1}}\left(S^{k} \mathscr{F}\right)_{y_{1}}$ et $\tilde{t}=0$ $\bmod \mathrm{m}_{y_{2}}\left(S^{k} \mathscr{F}\right)_{y_{2}}$. Si $t$ est la section de $\Gamma\left(X, \mathscr{L}^{k}\right)$ determinée par $\tilde{t}$, on aura $t\left(x_{1}\right)=$ $t_{1}\left(x_{1}\right) \neq 0$ et $t\left(x_{2}\right)=0$. Donc la restriction de $t$ à $T$ donne une section non triviale de $\mathcal{L}^{k}{ }_{\mid T}$, ce qui est absurde puisque ce faisceau est trivial et $T$ est compact irréductible. Donc $\operatorname{dim} Z=0$, et le Lemme 3.3 entraîne que $\mathcal{L}$ est ample et donc $\mathcal{F}$ est faiblement positif (Théorème 2.9).

LEMme 3.5. Soient $f: X \rightarrow Y$ un morphisme d'espaces analytiques compacts, $\&$ un faisceau inversible ample sur $X, \Phi \subset \vartheta_{Y}$ un idéal cohérent, $g=g, y$ un point de $Y$. Il existe alors deux entiers $k_{0}, n_{0}$ tels que

$$
\left(f_{*} g^{k} \complement^{n_{0} n}\right)_{y} \subset g_{y}\left(f_{*} \mathcal{L}^{n_{0} n}\right)_{y}
$$

pour tout $k \geqslant k_{0}$ et $n \geqslant 1$.

Remarquons que pour tout $n$ fixé il existe $k_{0}$ tel que $(*)$ soit vérifiée pour $k \geqslant k_{0}$ [8]; il faut montrer qu'on peut trouver un $k_{0}$ indépendant de $n$. L'algèbre $\vartheta_{Y} \oplus$ $\left(\oplus_{n>0} f_{*} \mathcal{L}^{n}\right)$ est de présentation finie $\operatorname{sur} \theta_{X}$ (voir [31, 2.1.2], ou la suite de cette démonstration), donc quitte à remplacer $\mathcal{L}$ par une de ses puissances on peut supposer qu'elle est engendrée au voisinage de $y$ par $f_{*} \mathcal{L}$. Soient $t_{1}, \ldots, t_{r}$ des générateurs $\left(t_{j} \in\left(f_{*} \mathcal{L}\right)_{y}\right)$, et $g_{1}, \ldots, g_{r}$ les éléments correspondants dans $\Gamma\left(f^{-1}(y), \mathcal{L}\right)$. Comme $\mathcal{L}_{\mid f^{-1}(y)}$ est ample, et donc $\mathcal{L}$ est relativement $f$-ample au voisinage de $f^{-1}(y)$ (Corollaire 2.10) on peut aussi supposer que $g_{1}, \ldots, g_{r}$ engendrent $\mathcal{L}_{x}$ pour tout $x \in f^{-1}(y)$. Soient en outre $u_{1}, \ldots, u_{p}$ de générateurs de $\mathscr{G}$ au 
voisinage de $y$, et $h_{1}, \ldots, h_{p}$ les éléments correspondants de $\Gamma\left(f^{-1}(y), g\right)$. Posons

$$
\mathfrak{K}=\theta_{X} \oplus\left[\bigoplus_{q \geqslant 1}\left(\bigoplus_{k+n=q} g^{k} \complement^{n}\right)\right] .
$$

$\mathrm{Au}$ voisinage de $f^{-1}(y)$ on peut donner à $\Re$ une structure de $\vartheta_{X}\left[T_{1}, \ldots, T_{p}, Z_{1}, \ldots, Z_{r}\right]$-module gradué, en faisant opérer $T_{j}$ au moyen de $h_{j}$, et $Z_{i}$ au moyen de $g_{i}$. Avec les techniques de [8] (cf. Démonstration du Lemme 2.4) on prouve que $\Re$ est un $\vartheta_{X}[T, Z]$-module gradué cohérent. D'après le Théorème 1 de [8] $f_{*}$ 的 est un $\vartheta_{Y}[T, Z]$-module cohérent. Soient $v_{1}, \ldots, v_{l}$ des générateurs de $\left(f_{*} \mathfrak{R}\right)_{y} \operatorname{sur} \theta_{Y, y}[T, Z]$. On peut supposer les $v_{j}$ homogènes, $v_{j} \in \Gamma\left(f^{-1}(y), g^{k_{j}} \varrho^{n_{j}}\right)$. Il suffit alors de prendre $k_{0}>\sup \left(k_{1}, \ldots, k_{l}\right)$ pour avoir le résultat.

Nous sommes maintenant en état de prouver le Théorème 3.1. En remplaçant $X$ par $\mathbf{P}(\mathcal{E})$, on se ramène tout de suite au cas où $\mathcal{E}$ un faisceau inversible $\mathcal{L}$. Nous montrons d'abord qu'il existe un entier $q_{0}$ tel que $f_{*} \mathcal{L}^{q_{0} m}$ est engendré par ses sections globales pour $m \geqslant 1$.

Soient $y \in Y, \mathscr{G}=\mathrm{m}_{y}$, et $k_{0}$ et $n_{0}$ les entiers donnés par le Lemme 3.5. Comme $\mathcal{L}$ est ample, on trouve que le morphisme naturel

$$
\Gamma\left(X, \varrho^{n_{0} m}\right) \rightarrow \Gamma\left(f^{-1}(y),\left\llcorner^{n_{0} m} / g^{k_{0}} \varrho^{n_{0} m}\right)\right.
$$

est surjectif pour tout $m$ assez grand; d'après le Lemme 3.5 on conclut que le morphisme $\Gamma\left(Y, f_{*} \mathcal{L}^{n_{0} m}\right)=\Gamma\left(X, \mathcal{L}^{n_{0} m}\right) \rightarrow\left(f_{*} \mathcal{L}^{n_{0} m}\right)_{y} / \mathfrak{m}_{y}\left(f_{*} \mathcal{L}^{n_{0} m}\right)_{y}$ est surjectif pour $m$ assez grand. Comme $X$ est compact, on trouve un entier $q_{0}$ tel que $f_{*} \mathcal{L}^{q_{0} m}$ est engendré par ses sections globales pour tout $m \geqslant 1$.

Soient $y_{1}, y_{2} \in Y$, et dénotons par $\mathfrak{m}_{y_{1} y_{2}}$ le faisceau des germes de section de $\theta_{Y}$ nulles en $y_{1}$ et $y_{2}$ si $y_{1} \neq y_{2}$, et nulles au moins du second ordre en $y$ si $y=y_{1}=y_{2}$. On utilise encore le Lemme 3.5 pour trouver qu'il existe $n_{1}$ tel que pour $m \geqslant 1$ l'application naturelle

$$
\Gamma\left(Y, f_{*} \mathcal{L}^{n_{1} m}\right) \rightarrow \Gamma\left(Y, f_{*} \mathcal{L}^{n_{1} m} \otimes \theta_{Y} / m_{y_{1} y_{2}}\right)
$$

est surjective.

Comme $(* *)$ reste surjective si l'on remplace $y_{1}$ et $y_{2}$ par des points suffisamment voisins et $X \times X$ est compact, on trouve $q_{1}$ tel que $f_{*} \sum^{q_{1}{ }^{n}}$ sépare les points de $Y$ pour $n \geqslant 1$. D'après le Lemme $3.4, f_{*} \varrho^{q_{0} q_{1} n}$ est faiblement positif pour tout $n \geqslant 1$, ce qui prouve le Théorème 3.1 .

Le Théorème 3.1 entraîne comme conséquence. ${ }^{1}$

ThÉRÈme 3.6. Soit $Y$ un espace complexe compact. Alors Y est de Moišezon si et seulement s'il existe sur $Y$ un faisceau faiblement positif de rang 1.

Preuve. Si $Y$ est un espace de Moišezon, il existe une modification propre $f$ : $X \rightarrow Y$ où $X$ est une variété algébrique projective. Soit $\mathcal{L}$ un faisceau inversible ample sur $X$; d'après le Théorème 3.1, pour $n$ convenable $f_{*} \mathcal{L}^{n}$ est faiblement positif sur $Y$.

Pour la réciproque voir par exemple $[2,4]$.

\footnotetext{
'Vo Van Tan m'a communiqué d'avoir une autre démonstration du Théorème 3.6.
} 
Le Théorème 3.1 permet aussi de donner une réponse affirmative à une conjecture de Grauert [12, p. 354].

THEOREME 3.7. Soient $Z$ un espace analytique, $Y$ un sous-espace compact rare de $Z$. Alors $Y$ est exceptionnel dans $Z$ si et seulement s'il existe un idéal $9 \subset \theta_{X}$, définissant $Y$, tel que $\mathrm{G} / \mathrm{G}^{2}$ soit un faisceau faiblement positif.

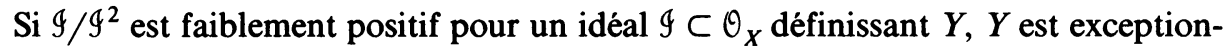
nel dans $Z$ d'après Grauert [12].

Réciproquement, supposons $Y$ exceptionnel dans $Z$, et soit $g: Z \rightarrow X$ une modification propre telle que $g(Y)=\{x\}, x \in X$, et que $g$ induise in isomorphisme de $Z \backslash Y$ sur $X \backslash\{x\}$. D'après le lemme de Chow analytique [17] (voir [3] pour le cas non réduit) il existe un diagramme commutatif

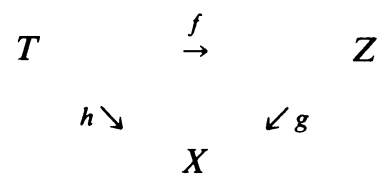

où $h$ est l'éclatement de $X$ de centre un idéal $g \subset \theta_{X}$ dont le support est $\{x\}$, et $f$ est l'éclatement de $Z$ de centre l'idéal $\mathcal{H}=\mathscr{G} \theta_{Z}$ (dont le support est $Y$ ). L'idéal $\mathcal{L}=\mathcal{H} O_{T}=\mathscr{G} O_{T}$ est inversible, et sa restriction à $h^{-1}(x)$ est un faisceau inversible ample. On a $f_{*} \mathcal{L}^{n}=\mathcal{H}^{n}$ pour tout $n$ assez grand. De la suite exacte

$$
0 \rightarrow \mathfrak{L}^{n+1} \rightarrow \mathfrak{L}^{n} \rightarrow \mathfrak{L}^{n} / \mathfrak{L}^{n+1} \rightarrow 0
$$

et du fait que $R^{1} f_{*} L^{n}$ est nul sur tout compact pour $n$ assez grand, on tire $f_{*}\left(\mathfrak{L}^{n} / \mathfrak{L}^{n+1}\right)=\mathcal{F}^{n} / \mathcal{F C}^{n+1}$ pour $n \gg 0$. D'après le Théorème 3.1 il existe un entier $q$ tel que $f_{*}\left[\left(\mathcal{L}^{n} / \complement^{n+1}\right)^{q}\right]=f_{*}\left(\complement^{n q} / \mathfrak{L}^{n q+1}\right)=\mathcal{F}^{n q} / \mathcal{F C}^{n q+1}$ est faiblement positif sur $Y$.

Soit $\mathscr{G}=\mathcal{H}^{n q}$. On peut considérer $g / \mathscr{G}^{2}$ comme un faisceau cohérent sur le sous-espace $Y_{g}$ de $Z$ défini par $\mathscr{G}_{\text {; }}$ sa restriction à l'espace $Y_{\mathscr{C}}$ défini par $\mathcal{H}$ est justement $\mathcal{H}^{n q} / \mathcal{F}^{n q+1}$. Il s'ensuit que $\mathscr{G} / \mathcal{G}^{2}$ est faiblement positif (2.7).

On peut établir les versions relatives des Théorèmes 3.1, 3.6. On laisse au lecteur le soin d'expliciter les démonstrations.

THEOREME 3.8. Soient

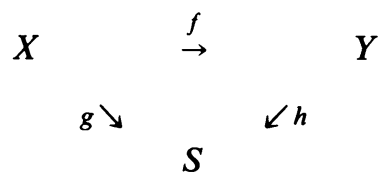

un diagramme commutatif de morphismes propres $d$ 'espaces analytiques, $\mathcal{E}$ un faisceau faiblement g-positif sur $X$. Pour tout ouvert relativement compact $U$ de $S$ il existe un entier positif $n_{0}$ tel que $f_{*}\left(S^{n_{0}} \mathcal{E}\right)$ soit faiblement $h$-positif au dessus de $U$ pour tout $n \geqslant 1$.

THÉRÈmE 3.9. Soit $h: Y \rightarrow S$ un morphisme propre d'espaces analytiques. Alors $Y$ est $h$-Moišezon en un point $s \in S$ si et seulement $s$ 'il existe un voisinage ouvert $U$ de $s$ dans $S$ et un faisceau faiblement h-positif de rang 1 sur $Y_{\mid S}$. 
4. Conditions pour l'existence des contractions. Le théorème suivant donne des conditions nécessaires et suffisantes pour l'existence des contractions analytiques. Il peut être considéré l'analogue relatif du Théorème 3.7.

Thtorème 4.1. Soient $X$ un espace analytique, $A$ un sous-espace fermé rare de $X$, $g \subset \vartheta_{X}$ un idéal définissant $A, f: A \rightarrow A^{\prime}$ un morphisme propre surjectif sur un autre espace analytique $A^{\prime}, \mathcal{E}=\mathscr{G} / \mathscr{G}^{2} \mid A, N=L(\mathcal{E})$. On suppose

(1) La restriction de $N$ à toute fibre de fest faiblement négative au sens de Grauert.

(2) Pour tout entier $n l$ 'application de faisceaux sur $A^{\prime}$ :

$$
f_{*}\left(\theta_{X} / g^{n}\right) \times_{f_{*} \theta_{A}} \theta_{A^{\prime}} \rightarrow \theta_{A^{\prime}}
$$

est surjective. Alors il existe une contraction de Xle long de $f$.

Réciproquement, si une telle contraction existe, on peut trouver (localement par rapport à $\left.A^{\prime}\right)$ un idéal $G \subset \vartheta_{X}$ définissant $A$ tel que les conditions (1) et (2) soient remplies.

Preuve. Si une contraction existe, la condition (2) est vérifiée pour tout idéal $G$ définissant $A$. Pour trouver un idéal $G$ vérifiant (1), on procède comme dans la preuve du Théorème 3.7 (on laisse les détails au lecteur).

On va prouver la réciproque. D'après le Corollaire 3.10, la condition (1) implique que $9 / \mathscr{G}^{2}$ est $f$-ample (localement par rapport à $\left.A^{\prime}\right)$. Soit $C=\operatorname{Specan}\left(\oplus_{n} g^{n} / g^{n+1}\right)$ le cône normal de $A$ dans $X$. On a un homomorphisme surjectif de $\theta_{X}$ algèbres $S\left(g / g^{2}\right) \rightarrow \oplus_{n} g^{n} / g^{n+1}$, d'où un plongement fermé de $C$ dans $L(\mathcal{E})$. D'après le Théorème 2.9 on peut contracter $L(\mathcal{E})$ le long de $f$, donc aussi $C$ (localement par rapport à $A^{\prime}$ ). Le résultat descend alors du corollaire du Théorème 2 de Fujiki [11]; une analyse de la démonstration de Fujiki montre en effet que la condition (ii) dans son théorème peut être remplacée par la condition (2) de notre énoncé.

5. Sur les différentes définitions de positivité. Il y a des nombreuses définitions de positivité pour un faisceau cohérent sur un espace analytique. Il semble qu'on puisse les grouper en deux types: celle de positivité faible de Grauert et ses variantes, et celle de Grauert-Riemenschneider [13] et ses variantes. Le problème de caractériser les espaces de Moišezon au moyen des faisceaux positifs au sens de GrauertRiemenschneider reste ouvert (voir $[\mathbf{2 4}, \mathbf{2 5}, \mathbf{2 8}, \mathbf{2 9}$ ] pour des cas particuliers importants). Remarquons que, dans ce cas, la partie difficile consiste à prouver qu'un espace qui porte un faisceau positif est de Moišezon, la réciproque étant triviale.

Une étude détaillée des notions de positivité du premier type a été faite par Rabinowitz [22, 23]; en particulier, il démontre dans [22] le Corollaire 2.11 (dans le cas d'un espace compact normal et irréductible) et il annonce dans [23] le Théorème 3.6 dans le cas d'une surface normale irréductible.

Nous pouvons ici, au moyen du Théorème 3.1, préciser les rapports entre les faisceaux faiblement positifs et les faisceaux primairement faiblement positif au sens de [22].

Pour tout faisceau cohérent $\mathscr{F}$ notons $\mathscr{F}_{\#}$ le quotient de $\mathscr{F}$ par son sous-faisceau de torsion. On a 
ThÉrème 5.1. Soient $X$ un espace analytique compact, $\mathscr{F}$ un faisceau cohérent sur $X$. $S i \mathcal{F}$ est primairement faiblement positif, il existe un entier $n$ tel que $\left(S^{n} \mathcal{F}\right)_{\#}$ soit faiblement positif.

On peut supposer $X=\operatorname{Supp} \mathscr{F}$. D'après [22] il existe un espace analytique compact $P$, une projection surjective $p: P \rightarrow X$, un faisceau inversible ample $\mathcal{H}$ sur $P$, tel que $p_{*} \mathcal{F}^{n}=\left(S^{n} \mathscr{F}\right)_{\#}$ pour tout $n$ assez grand. Le théorème est alors une conséquence du Théorème 3.1.

ADDED IN PROOF. T. Peternell a publié (Manuscripta Math. 37 (1982), 19-26) une preuve des Théorèmes 3.6 et 3.7. Mais la preuve de son Satz 3 (p. 21) ne semble pas être correcte; en effet (avec ses notations) en général on n'a pas $\tilde{A}_{\text {red }}=P\left(\mathfrak{S} / \mathfrak{\Im}^{2}\right)_{\text {red }}$ (l'égalité implique, par exemple, que les fibres réduites de l'éclatement $\sigma$ aux points de $A$ sont des espaces projectifs, ce qui n'est pas toujours le cas).

\section{BIBLIOGRAPHIE}

1. V. Ancona, Un teorema di contrattibilità relativa, Boll. Un. Mat. Ital. A (5) 9 (1974), 785-790.

2. Espaces fibrés linéaires faiblement négatifs sur un espace complexe, Trans Amer. Math. Soc. 215 (1976), 45-61.

3. Espaces de Moišezon relatifs et algébrisation des modifications analytiques, Math. Ann. 246 (1980), 155-165.

4. V. Ancona et Vo Van Tan, Embedding Moishezon spaces into 1-convex spaces, Math. Ann. 247 (1980), 143-147.

5. A. Andreotti et W. Stoll, Analytic and algebraic dependence of meromorphic functions, Lecture Notes in Math., vol. 234, Springer-Verlag, Berlin, Heidelberg and New York, 1971.

6. M. Artin, The implicit function theorem in algebraic geometry, Algebraic Geometry, Oxford Univ. Press, London, 1969.

7. Algebraisation of formal moduli. II. Existence of modifications, Ann. of Math. (2) 91 (1970), $88-135$.

8. C. Banica, Le complété formel d'un espace analytique le long d'un sous-espace: un théorème de comparaison, Manuscripta Math. 6 (1972), 207-244.

9. J. Bingener, Schemata über Steinschen algebren, Schr. Math. Inst. Univ. Münster (2) 10 (1976).

10. G. Fischer, Lineare Faserraüme und kohärente Modulgarben über komplexen Räumen, Arch. Math. (Basel) 18 (1967), 609-617.

11. A. Fujiki, On the blowing down of analytic spaces, Publ. Res. Inst. Math. Sci. 10 (1975), 473-507.

12. H. Grauert, Über Modifikationen und exzeptionelle analytische Mengen, Math. Ann. 146 (1962), $331-368$.

13. H. Grauert et O. Riemenschneider, Verschwindungssätze für analytische Kohomologiegruppen auf komplexen Räumen, Invent. Math. 11 (1970), 263-292.

14. A. Grothendieck et J. Dieudonné, Eléments de géométrie algébrique. II, III, Inst. Hautes Études Sci. Publ. Math., Paris, 1961, pp. 8-11.

15. M. Hakim, Topos annelés et schémas relatifs, Springer-Verlag, Berlin, Heidelberg and New York, 1972.

16. R. Hartshorne, Ample vector bundles, Inst. Hautes Études Sci. Publ. Math. 29 (1966), 319-350.

17. H. Hironaka, Flattening theorem in complex analytic geometry, Amer. J. Math. 97 (1975), 503-547.

18. K. Knorr et M. Schneider, Relativexzeptionelle analytische Mengen, Math. Ann. 193 (1971), 238-254.

19. D. Knutson, Algebraic spaces, Lecture Notes in Math., vol. 203, Springer-Verlag, Berlin, Heidelberg and New York, 1971.

20. N. Kuhlmann, Über holomorphe Abbildungen mit proiektiven Fasern, Math. Z. 135 (1973), 43-54.

21. J. Mazur, Conditions for the existence of contractions in the category of algebraic spaces, Trans. Amer. Math. Soc. 209 (1975), 259-265.

22. J. H. Rabinowitz, Positivity notions for coherent sheaves over compact complex spaces, Invent. Math 62 (1980), 79-87. 
23. (1981), $121-133$.

Finsler structures of negative curvature in C-linear fibre spaces, Manuscripta Math. 34

24. O. Riemenschneider, Characterizing Moišezon spaces by almost positive coherent analytic sheaves, Math. Z. 123 (1971), 263-284.

25. , A generalization of Kodaira's embedding theorem, Math. Ann. 200 (1973), 99-102.

26. M. Schneider, Familien negativer Vektorraümbundel und 1-konvexe Abbildungen, Abh. Math. Sem. Univ. Hamburg 47 (1978), 150-170.

27. Séminaire H. Cartan 1960-61, Exposé XII par A. Grothendieck, Fibrés vectoriels, fibrés projectifs, fibrés en drapeaux. Familles d'espaces complexes et fondements de la géométrie analytique, Ann. Sci. École. Norm. Sup., Paris, 1962.

28. A. J. Sommese, Criteria for quasi-projectivity, Math. Ann. 217 (1975), 247-256.

29. __ Addendum to "Criteria for quasi-projectivity", Math. Ann. 221 (1976), 95-96.

30. __ Submanifolds of abelian varieties, Math. Ann. 233 (1978), 229-256.

31. G. Tomassini, Modifications des espaces complexes. I, Ann. Mat. Pura Appl. (4) 103 (1975), 369-395.

32. F. L. Zak, q-finite morphisms in formal algebraic geometry, Izv. Akad. Nauk SSSR Ser. Mat. 39 (1975), 28-68; Traduit en anglais dans Math. USSR-Izv. 9 (1975), 27-62.

Istituto Matematico, Universita di Firenze, Viale Morgagni 67/A, 50134 Firenze, Italy 